\title{
Phonetic notation
}

Phonetic notation will follow the International Phonetic Alphabet (IPA), as shown in Figure 0.1. Readers are assumed to be familiar with phonetic terms (a good introduction is Johnson 2003).

\section{ENGLISH VOWEL NOTATION}

Because English vowels are disproportionately important in the field of linguistic variation, it is worth mentioning the notation adopted for them in this book. In general, I use the IPA vowel that most closely approximates the vowel class. I generally gloss each with an example word. However, because of differences in the pronunciation of vowels in different varieties of English, it is inevitable that a speaker's native pronunciation (or the primary variety learned, if English is a second language) will differ in some details. This variety of vowel classes is also the reason for not using the keywords of Wells (1982). Here are the general representations.
$/ \alpha /$ lot
/O/ thought
/o/ above
/E/ bet
/el bait
/I/ bit
/i/ beet
|æl bat
/u/ boot
/u/ foot
/v/ boat

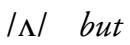
lau/ house
laI/ bite
lou/ boat
lo1/ boy 
THE INTERNATIONAL PHONETIC ALPHABET (revised to 2005)

CONSONANTS (PULMONIC)

\begin{tabular}{|c|c|c|c|c|c|c|c|c|c|c|c|}
\hline & Bilabial & Labiodent & Dental & Alveolar & Postalveolar & Retroflex & Palatal & Velar & Uvular & Pharyngeal & Glottal \\
\hline Plosive & $\mathrm{p} \quad \mathrm{b}$ & & & $\mathrm{t} \mathrm{d}$ & & t d & c $\mathrm{f}$ & $\mathrm{kg}$ & $q \quad G$ & & $?$ \\
\hline Nasal & $\mathrm{m}$ & $\mathrm{m}$ & & $\mathrm{n}$ & & $\eta$ & $\mathrm{n}$ & $\eta$ & $\mathrm{N}$ & & \\
\hline Trill & B & & & $\mathrm{r}$ & & & & & $\mathrm{R}$ & & \\
\hline Tap or Flap & & V & & r & & $\mathrm{r}$ & & & & & \\
\hline Fricative & $\phi \beta$ & f $\quad v$ & $\theta \quad \partial$ & $S \quad Z$ & $\int 3$ & $\begin{array}{ll}S & Z_{4}\end{array}$ & Ç J & $x \quad \gamma$ & $\chi$ в & h $\mathrm{S}$ & h h \\
\hline $\begin{array}{l}\text { Lateral } \\
\text { fricative } \\
\end{array}$ & & & & 13 & & & & & & & \\
\hline Approximant & & $v$ & & I & & t & $\mathrm{j}$ & щu & & & \\
\hline \begin{tabular}{|l|}
$\begin{array}{l}\text { Lateral } \\
\text { approximant }\end{array}$ \\
\end{tabular} & & & & 1 & & 1 & $\Lambda$ & $\mathrm{L}$ & & & \\
\hline
\end{tabular}

Where symbols appear in pairs, the one to the right represents a voiced consonant. Shaded areas denote articulations judged impossible.

CONSONANTS (NON-PULMONIC)

\begin{tabular}{|c|c|c|c|c|}
\hline \multicolumn{2}{|r|}{ Clicks } & Voiced implosives & \multicolumn{2}{|r|}{ Ejectives } \\
\hline$\odot$ & Bilabial & 6 Bilabial & , & Examples: \\
\hline & Dental & d Dental/alveolar & $\mathrm{p}^{\prime}$ & Bilabial \\
\hline$!$ & (Post)alveolar & $f$ Palatal & t' & Dental/alveolar \\
\hline$\neq$ & Palatoalveolar & @ velar & $\mathrm{k}^{\prime}$ & Velar \\
\hline$\|$ & Alveolar lateral & G Uvular & $\mathrm{S}^{\prime}$ & Alveolar fricative \\
\hline
\end{tabular}
M Voiceless labial-velar fricative
$6 \mathrm{Z}$ Alveolo-palatal fricatives
W Voiced labial-velar approximant
I voiced alveolar lateral flap
U Voiced labial-palatal approximant
H Voiceless epiglottal fricative
£ Voiced epiglottal fricative
f Epiglottal plosive
Affricates and double articulations
can be represented by two symbol joined by a tie bar if necessary.

kp ts

DIACRITICS Diacritics may be placed above a symbol with a descender, e.g. $\stackrel{\circ}{\text { ] }}$

\begin{tabular}{|c|c|c|c|c|c|}
\hline$\circ$ & Voiceless & $n_{0} \mathrm{~d}_{0}$ & .. & $\begin{array}{lll}\text { Breathy voiced } & \text { b } & \stackrel{.}{a}\end{array}$ & $\underset{n}{\mathrm{t}} \mathrm{d}$ \\
\hline$\checkmark$ & Voiced & $S \quad t$ & $\sim$ & Creaky voiced $\underset{\sim}{\mathrm{b}} \underset{\sim}{\mathrm{a}}$ & $\underset{s}{\mathrm{~d}}$ \\
\hline $\mathrm{h}$ & Aspirated & $\mathrm{t}^{\mathrm{h}} \mathrm{d}^{\mathrm{h}}$ & $\sim$ & Linguolabial $\quad \underset{\sim}{\mathrm{t}} \quad \underset{\sim}{\mathrm{d}}$ & _ Laminal \\
\hline , & More rounded & วิ & $\mathrm{W}$ & Labialized $\quad \mathrm{t}^{\mathrm{W}} \mathrm{d}^{\mathrm{W}}$ & $\sim \quad$ Nasalized \\
\hline$c$ & Less rounded & ?ִ & $\mathrm{j}$ & Palatalized $\quad \mathrm{t}^{\mathrm{j}} \quad \mathrm{d}^{\mathrm{j}}$ & $\mathrm{n}$ Nasal release \\
\hline+ & Advanced & $\bigcup_{+}$ & $\mathrm{Y}$ & Velarized $\quad t^{\gamma} d^{\gamma}$ & $1 \quad$ Lateral release \\
\hline- & Retracted & $\underline{e}$ & \$ & Pharyngealized $\mathrm{t}^{\mathrm{S}} \mathrm{d}^{\mathrm{S}}$ & $7 \quad$ No audible release $\quad \mathrm{d}^{\urcorner}$ \\
\hline$\ddot{*}$ & Centralized & $\ddot{\mathrm{e}}$ & $\sim$ & Velarized or pharyngealized $\mathbf{f}$ & \\
\hline$x$ & Mid-centralized & $\stackrel{x}{e}$ & $\perp$ & Raised & $=$ voiced alveolar fricative) \\
\hline & Syllabic & $\mathrm{n}_{1}$ & $T$ & Lowered & $=$ voiced bilabial approximant) \\
\hline n & Non-syllabic & $\mathrm{e}$ & -1 & Advanced Tongue Root & \\
\hline$\imath$ & Rhoticity & $\partial^{r} a^{r}$ & & Retracted Tongue Root & \\
\hline
\end{tabular}

VOWELS
OTHER SYMBOLS

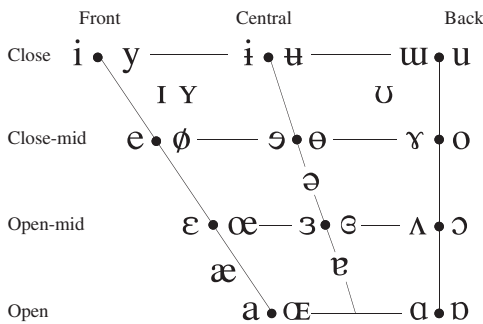

Where symbols appear in pairs, the one to the right represents a rounded vowel.

SUPRASEGMENTALS

1 Primary stress

I Secondary stress

,founo'tifon

I Long e:

- Half-long $e^{r}$

$\checkmark$ Extra-short $\breve{\mathrm{e}}$

Minor (foot) group

I| Major (intonation) group

- Syllable break Ii.æekt

$\smile \quad$ Linking (absence of a break)

TONES AND WORD ACCENTS

LEVEL AONTOUR

$\left.\ddot{e}_{\text {or }}\right\rceil \begin{aligned} & \text { Extra } \\ & \text { high }\end{aligned} \check{e}_{\text {or }} \Lambda$ Rising

é $\dashv$ High $\hat{\mathrm{V}} \mathrm{V}$ Falling

e $\dashv \mathrm{Mid}$

è $\dashv$ Low

Extr

è $」 \begin{gathered}\text { Extra } \\ \text { low }\end{gathered}$

$\downarrow \quad$ Downstep

$\uparrow \quad$ Upstep

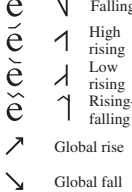

Figure 0.1 International Phonetic Alphabet (copyright International Phonetic Association) 